\title{
Positron emission tomography/computed tomography for bone tumors (Review)
}

\author{
AIKEREMUJIANG MUHEREMU and XIAOHUI NIU \\ Department of Orthopedic Oncology Surgery, Beijing JiShui Tan Hospital, Beijing 100035, P.R. China
}

Received February 9, 2014; Accepted August 22, 2014

DOI: $10.3892 / \mathrm{ol} .2014 .2728$

\begin{abstract}
The aim of the present study was to investigate positron emission tomography (PET)/computed tomography (CT) and its applications for the diagnosis and treatment of bone tumors. The advantages and disadvantages of PET/CT were also evaluated and compared with other imaging methods and the prospects of PET/CT were discussed. The PubMed, Medline, Elsevier, Wanfang and China International Knowledge Infrastructure databases were searched for studies published between 1995 and 2013, using the terms 'PET/CT', 'positron emission tomography', 'bone tumor', 'osteosarcoma', 'giant cell bone tumor' and 'Ewing sarcoma'. All the relevant information was extracted and analyzed. A total of 73 studies were selected for the final analysis. The extracted information indicated that at present, PET/CT is the imaging method that exhibits the highest sensitivity, specificity and accuracy. Although difficulties and problems remain to be solved, $\mathrm{PET} / \mathrm{CT}$ is a promising non-invasive method for the diagnostic evaluation of and clinical guidance for bone tumors.
\end{abstract}

\section{Contents}

1. Introduction

2. Mechanisms of PET/CT

3. Current applications of PET/CT

4. Comparison of PET/CT and other imaging methods

5. Problems and prospective of PET/CT

6. Conclusion

Correspondence to: $\mathrm{Dr}$ Xiaohui Niu, Department of Orthopedic Oncology Surgery, Beijing JiShui Tan Hospital, 31 Xinjiekou East Street, Beijing 100035, P.R. China

E-mail: niuxiaohui@263.net

Key words: positron emission tomography/computed tomography, imaging, bone tumor

\section{Introduction}

Diagnostic imaging is of great importance for the initial staging and assessment of recurrence of various malignancies. The differentiation of benign and malignant intraosseous lesions may often be achieved using radiographical methods, including X-ray, computed tomography (CT) and magnetic resonance imaging (MRI) (1). Radiographs, including CT and $\mathrm{X}$-ray, provide important information regarding the appearance, intraosseous extent and internal characteristics of bone tumors (1).

Positron emission tomography (PET) using 2-(fluorine-18)-2-deoxy-D-glucose $\left({ }^{18} \mathrm{~F}-\mathrm{FDG}\right)$ has been used to differentiate malignant tumors from benign lesions (2). In the musculoskeletal system, findings of previous studies have shown a positive correlation between glucose consumption measured by ${ }^{18} \mathrm{~F}$-FDG and the aggressiveness of musculoskeletal tumors (3). Griffeth etal (4) performed ${ }^{18} \mathrm{~F}$-FDG PET studies on a series of 20 intraosseous lesions, where 14/15 malignant tumors and 4/5 benign lesions were correctly diagnosed, using a cut-off value of 2.0 for the maximum standardized uptake volume $\left(\mathrm{SUV}_{\max }\right)$. However, $13 / 15$ of the malignant tumors were metastatic carcinomas. Aoki et al (5) identified a statistically significant difference between the $\mathrm{SUV}_{\max }$ values of benign and malignant bone tumors in 52 bone lesions. However, the authors observed a significant overlap between the $\mathrm{SUV}_{\max }$ values of a number of the benign and malignant bone tumors, and a high accumulation of ${ }^{18} \mathrm{~F}$-FDG was reported in certain benign bone tumors. However, other studies have questioned the correlation between ${ }^{18} \mathrm{~F}-\mathrm{FDG}$ accumulation and the malignant potential of bone tumors. Furthermore, the value of ${ }^{18} \mathrm{~F}$-FDG in the musculoskeletal system has also been questioned. The intensity of ${ }^{18} \mathrm{~F}$-fluorodeoxyglucose (FDG) uptake has been found to positively correlate with the histological grade of various malignancies. The combined metabolic and morphological information provided by the FDG PET/CT imaging has been shown to exhibit high sensitivity for initial staging and assessment of recurrence of soft tissue and osseous sarcomas in retrospective studies (6).

Dual-time point ${ }^{18} \mathrm{~F}$-FDG PET is practical and may be useful for differentiating malignant tumors from benign ones (7). Serial FDG-PET imaging accurately classified histopathological response/non-response to neoadjuvant therapy in adult primary bone sarcomas. Notably, a combination of previously applied response criteria (reductions 
Table I. Summary of previous studies using positron emission tomography/computed tomography for the diagnosis of musculoskeletal tumors.

\begin{tabular}{|c|c|c|c|c|c|c|c|}
\hline \multirow[b]{2}{*}{ Study, year (Refs.) } & \multirow{2}{*}{$\begin{array}{l}\text { No. of } \\
\text { patients }\end{array}$} & \multicolumn{2}{|c|}{$\mathrm{SUV}_{\max }$} & \multirow{2}{*}{$\begin{array}{l}\mathrm{SUV}_{\text {max }} \\
\text { cut off }\end{array}$} & \multirow{2}{*}{$\begin{array}{c}\text { Sensitivity, } \\
\%\end{array}$} & \multirow{2}{*}{$\begin{array}{c}\text { Specificity, } \\
\%\end{array}$} & \multirow{2}{*}{$\begin{array}{c}\text { Accuracy, } \\
\%\end{array}$} \\
\hline & & Benign & Malignant & & & & \\
\hline Fuglø 2012 (9) & 89 & & & & 95.8 & 92.7 & 93.1 \\
\hline Aoki 2003 (5) & 114 & $\begin{array}{c}1.80 \\
(\mathrm{SD}, \pm 1.42)\end{array}$ & $\begin{array}{c}4.20 \\
(\mathrm{SD}, \pm 3.16)\end{array}$ & & & & \\
\hline Shin 2008 (10) & 34 & $\begin{array}{c}2.9 \\
\text { (range, 0.6-5.5) }\end{array}$ & $\begin{array}{c}12.0 \\
\text { (range, } 4.3-45.7)\end{array}$ & 4.7 & 89.6 & 86.7 & 88.2 \\
\hline Piperkova 2008 (11) & 93 & & & & 100 & 95.9 & \\
\hline Charest 2009 (6) & 212 & & & & & 93.9 & \\
\hline Iagaru 2006 (12) & 44 & & & & 100 & 93.3 & \\
\hline Strobel (13) & 50 & $\begin{array}{c}3.5 \\
\text { (range, 1.6-8.0) }\end{array}$ & $\begin{array}{c}3.5(1.6-8.0) \\
\text { (range, } 1.6-8.0)\end{array}$ & & 91 & 77 & 86 \\
\hline Shin 2008 (14) & 87 & 5.1 & 8.8 & 4.7 & 80 & 63 & 70 \\
\hline Eary $2002(15)$ & 209 & & $\begin{array}{c}6 \\
(\text { range, } 3.8-11.3)\end{array}$ & & & & \\
\hline
\end{tabular}

$\mathrm{SD}$, standard deviation; $\mathrm{SUV}_{\max }$, maximum standardized uptake volume.

in $\mathrm{SUV}_{\max }$ by $\geq 60 \%$ and a post-treatment tumor $\mathrm{SUV}_{\max }$ of $<2.5)$ provided the optimal response predictions (8).

\section{Mechanisms of PET/CT}

FDG PET and CT are imaging methods that have been found to be valuable in clinical application. Integrated PET/CT, which uses a combination of PET and CT in one imaging device, performs morphological and functional imaging in one procedure. Integrated PET/CT has been shown to be more accurate for lesion localization and characterization than PET and CT alone and the results obtained from PET and CT separately, following software-based fusion of the PET and CT data sets (16).

\section{Current applications of PET/CT}

Diagnosis of bone tumor malignancy. ${ }^{18} \mathrm{~F}-\mathrm{FDG}$ PET enables the assessment of glucose metabolism and thus the metabolic activity of cancer tissue by calculating a SUV value (Table I). At present, ${ }^{18} \mathrm{~F}$-FDG PET is successfully used and approved for procedure reimbursement in numerous cancer types, including lung cancer, melanoma, lymphoma, head and neck tumors, brain tumors, esophageal cancer and colorectal cancer (17).

Osteosarcoma. Osteosarcoma is the most common primary malignancy of the bone, with the peak age of incidence ranging between 10 and 25 years (18). The initial stage and necrosis of tumors are the clearest prognostic factors (19). Osteosarcoma typically recurs within 2-3 years of surgery (20). Therefore, a longer follow-up period is required for a more complete assessment of survival. In a study by Cistaro et al (21), including 18 children with osteosarcoma and Ewing sarcoma, it was demonstrated that ${ }^{18} \mathrm{~F}-\mathrm{FDG}$ PET/CT may be used to identify the metabolic characteristics of lung nodules in patients with bone sarcoma. In addition, a $\mathrm{SUV}_{\max }$ (or SUV ratio of $>1$ ) was identified as a sensitive and specific marker to assess whether a lesion with a diameter of $<6 \mathrm{~mm}$ is malignant or benign (22).

However, not all cancer types may be detected by PET/CT with high accuracy. Variable uptake is considered to be associated with the biological features of individual cancers, which has been observed in bronchoalveolar carcinomas, renal and thyroid cancers, several subtypes of malignant lymphoma and carcinoids, as well as the majority of prostate carcinomas. The prognostic relevance of this biological heterogeneity is not clear. However, in the majority of cases, FDG PET is a sensitive imaging modality for the detection, staging and re-staging, as well as the assessment of therapy response in cancers (23-25).

Findings of previous studies have shown that delayed PET (2-3 $\mathrm{h}$ following injection) may aid with the differentiation of malignant lesions from benign lesions $(26,27)$. Sahlmann et al (28) investigated glucose metabolism in 17 patients with chronic bacterial osteomyelitis and four patients with malignant bone lesions using a dual time point ${ }^{18} \mathrm{~F}-\mathrm{FDG}$ PET (30 and 90 min following injection). The authors hypothesized that dual time point ${ }^{18} \mathrm{~F}$-FDG PET may be of value for the differentiation of chronic bacterial osteomyelitis and malignant bone lesions. However, the sample size of the study was small and only one type of benign bone lesion was investigated.

Detection of cancer metastases. FDG PET has been shown to be useful for the detection of nodal and distant metastases in patients with soft tissue sarcomas when compared with that of conventional imaging (29). We hypothesized that PET/CT may also be an effective method for this purpose. In a study by Klaeser et al (30), 12 patients who were admitted to hospital for staging and restaging of breast cancer, non-small 
cell lung cancer, cervical cancer, soft tissue sarcoma and osteosarcoma were repositioned according to the results of PET-CT. Subsequently, intervention was planned according to a single-bed PET-CT acquisition of the affected region. The metabolically active sections of the lesions were accurately targeted in all the patients and representative samples were obtained in $92 \%$ of patients (30).

Responses to therapeutic interventions. Changes in SUV following neoadjuvant chemotherapy (NACT) have been reported to be useful for the prediction of tumor response in osteosarcoma (31). The standard chemotherapy response evaluation used currently histologically assesses the tumor necrosis of the excised lesion (32), which has been reported to be the most important prognostic factor in osteosarcoma following NACT (33). However, as tumor necrosis may only be assessed in the resected specimens by surgery or biopsy, the continuation of ineffective chemotherapy may result in tumor resistance. ${ }^{18} \mathrm{~F}-\mathrm{FDG}$-PET-CT may serve as a surrogate for histological necrosis and may decrease the time required to evaluate the efficacy of NACT to determine the optimal therapeutic intervention (34).

The use of ${ }^{18} \mathrm{~F}-\mathrm{FDG}$ PET was previously for the evaluation of osteosarcoma response to NACT $(35,36)$. The association between tumor necrosis and outcome, as well as the association between worse survival and high metabolic activity in chemotherapy-naive tumors and high metabolic activity following chemotherapy, provide similar conclusions to those of previous studies, whereby ${ }^{18} \mathrm{~F}-\mathrm{FDG}$ PET accurately reflects osteosarcoma response to chemotherapy. Despite the small number of mortalities and short follow-up period, these results are similar to those that indicate that survival is associated with $\mathrm{SUV}_{\max }$ following chemotherapy (36).

The ${ }^{18} \mathrm{~F}-\mathrm{FDG}$ uptake of the majority of pediatric sarcomas is high, indicating that ${ }^{18} \mathrm{~F}-\mathrm{FDG}$ PET/CT imaging may be used to monitor responses to therapeutic interventions (37).

Prediction of prognosis. Costelloe et al (38) analyzed the $\mathrm{SUV}_{\text {max }}$ of 33 patients with osteosarcoma using ${ }^{18} \mathrm{~F}-\mathrm{FDG}$ positron-emission tomography-computed tomography (PET/CT) and found that although the location of high $\mathrm{SUV}_{\max }$ values was not associated with the five-year survival rate of patients, a high $\mathrm{SUV}_{\text {max }}$ value was significantly associated with a low five-year survival rate of patients (38).

\section{Comparison of PET/CT and other imaging modalities}

Bone scans. Although few studies have compared the diagnostic accuracy of ${ }^{18} \mathrm{~F}$-FDG PET with that of bone scans, ${ }^{18} \mathrm{~F}$-FDG PET has demonstrated a higher sensitivity than that of bone scans for the detection of bone or bone marrow metastases. The detection rates of ${ }^{18} \mathrm{~F}$-FDG PET in previous studies were $80-100 \%(39,40)$, while the detection rates of bone scans were 61 and 75\% (39).

MRI. MRI is an extremely sensitive method for the detection of bone marrow abnormalities, cortical destruction or soft tissue tumors adjacent to or infiltrating neighboring bone (40). However, the morphological appearance of numerous lesions is non-specific. With certain entities, the diagnosis may be difficult with radiographical imaging or MRI.
PET and CT. Antoch et al (41) compared the value of PET, CT and dual-modality PET/CT imaging for assessing gastro-intestinal stromal tumor (GIST) response to imatinib therapy and demonstrated that image fusion with combined PET/CT may provide additional information in individual cases when compared with side-by-side PET and CT (41).

Although tumor size and infiltration of adjacent structures may be accurately assessed using CT (42), PET has been demonstrated to be markedly more sensitive and specific in the detection and characterization of metastases to mediastinal lymph nodes (43). The limited anatomical information yielded by PET may be overcome by combining the functional PET data with morphological CT data.

In a study by Yi et al (44), which included 119 patients, the sensitivity and accuracy of PET/CT for the accuracy for pulmonary nodule characterization was investigated and was found to be significantly higher than that of helical dynamic CT.

Regarding the combination of PET/CT and conventional imaging, the accuracy of $\mathrm{T}$ staging for osteosarcoma was significantly more accurate than PET alone. However no significant differences were identified between conventional methods or PET/CT. PET/CT is significantly more accurate for the N and $\mathrm{M}$ staging of osteosarcoma than conventional imaging methods $(\mathrm{P}<0.01)$. These results indicated that the combination of PET/CT and conventional imaging is the optimal preoperative staging method for bone and soft tissue sarcomas, due to its significantly higher diagnostic accuracy (45).

\section{Problems and prospects for PET/CT}

Although PET/CT has been found to be useful in the diagnosis and treatment of musculoskeletal tumors, a number of issues remain that require further study. Although these issues remain a challenge, they may present potential opportunities for the wide application of PET/CT in orthopedic oncology.

Application of $S U V$. In two previous studies, $\mathrm{SUV}_{\max }$ was used as a parameter for response prediction $(46,47)$. In these studies, SUV2/SUV1 and SUV2 were found to correlate with histological response. In addition, an SUV2 of $<2$ and an SUV2/SUV1 of $<0.4$ were accurate response indicators.

Application of tumor volume measurements. Tumor volume measurements are easier to obtain and more reproducible in osteosarcoma than in other tumor types, when an appropriate MRI technique is used (48).

Combined PET/CT and MRI systems. Combined PET/MRI systems allow the simultaneous or sequential acquisition of PET and MRI images (49), and literature regarding previous studies and the initial clinical experiences with PET/MRI in oncology are available (50).

Combined metabolic and volumetric information on ${ }^{18}$ F-FDG PET and MRI scans, obtained prior to and following chemotherapy completion, may be used to predict the histological response to NACT in osteosarcoma (51).

For specific tumors that are not PET/CT sensitive, the combined use of PET/CT and MRI may significantly increase the sensitivity of diagnosis (52). 
Accurate anatomic localization of functional abnormalities observed with PET is known to be difficult. Although non-specific tracers, such as ${ }^{18} \mathrm{~F}-\mathrm{FDG}$, visualize certain normal anatomical structures, the spatial resolution is generally inadequate for the localization of pathology. Combining PET with a high-resolution anatomical imaging modality, such as CT, may resolve this localization issue. However, the images captured from the two modalities must be accurately coregistered.

\section{Conclusion}

The combined metabolic and morphological information provided by FDG PET/CT imaging provides sensitivity for initial staging and the assessment of recurrence of soft tissue and osseous sarcomas.

\section{References}

1. Nishio J, Ideta S, Iwasaki H and Naito M: Scapular osteochondrolipoma: Imaging features with pathological correlation. Oncol Lett 6: 817-820, 2013.

2. Hillner BE, Siegel BA, Shields AF, et al: Relationship between cancer type and impact of PET and PET/CT on intended management: findings of the national oncologic PET registry. $\mathrm{J}$ Nucl Med 49: 1928-1935, 2008.

3. Dimitrakopoulou-Strauss A, Strauss LG, Heichel T, et al: The role of quantitative (18)F-FDG PET studies for the differentiation of malignant and benign bone lesions. J Nucl Med 43: 510-518, 2002.

4. Griffeth LK, Dehdashti F, McGuire AH, et al: PET evaluation of soft-tissue masses with fluorine-18 fluoro-2-deoxy-D-glucose. Radiology 182: 185-194, 1992

5. Aoki J, Watanabe H, Shinozaki T, et al: FDG-PET for preoperative differential diagnosis between benign and malignant soft tissue masses. Skeletal Radiol 32: 133-138, 2003.

6. Charest M, Hickeson M, Lisbona R, et al: FDG PET/CT imaging in primary osseous and soft tissue sarcomas: a retrospective review of 212 cases. Eur J Nucl Med Mol Imaging 36: 1944-1951, 2009.

7. Tian R, Su M, Tian Y, et al: Dual-time point PET/CT with F-18 FDG for the differentiation of malignant and benign bone lesions. Skeletal Radiol 38: 451-458, 2009.

8. Benz MR, Czernin J, Tap WD, et al: FDG-PET/CT Imaging Predicts Histopathologic Treatment Responses after Neoadjuvant Therapy in Adult Primary Bone Sarcomas. Sarcoma 2010: doi:10.1155/2010/143540.

9. Fugl $\varnothing$ HM, Jørgensen SM, Loft A, Hovgaard D and Petersen MM: The diagnostic and prognostic value of ${ }^{18} \mathrm{~F}-\mathrm{FDG}$ PET/CT in the initial assessment of high-grade bone and soft tissue sarcoma. A retrospective study of 89 patients. Eur J Nucl Med Mol Imaging 39: 1416-1424, 2012.

10. Shin DS, Shon OJ, Byun SJ, et al: Differentiation between malignant and benign pathologic fractures with F-18-fluoro-2-deoxy-D-glucose positronemission tomography/ computed tomography. Skeletal Radiol 37: 415-421, 2008.

11. Piperkova E, Mikhaeil M, Mousavi A, et al: Impact of PET and $\mathrm{CT}$ in PET/CT studies for staging and evaluating treatment response in bone and soft tissue sarcomas. Clin Nucl Med 34: 146-150, 2009

12. Iagaru A, Quon A, McDougall IR and Gambhir SS: F-18 FDG PET/CT evaluation of osseous and soft tissue sarcomas. Clin Nucl Med 31: 754-760, 2006.

13. Strobel K, Exner UE, Stumpe KD, et al: The additional value of CT images interpretation the differential diagnosis of benign vs. malignant primary bone lesions with 18F-FDG -PET/CT. Eur J Nucl Med Mol Imaging 35: 2000-2008, 2008.

14. Shin DS, Shon OJ, Hans DS, Choi JH, Chun KA and Cho IH: The clinical efficacy of (18)F-FDG-PET/CT in benign and malignant musculoskeletal tumors. Ann Nucl Med 22: 603-609, 2008.

15. Eary JF, O'Sullivan F, Powitan Y, et al: Sarcoma tumor FDG uptake measured by PET and patient outcome: a retrospective analysis. Eur J Nucl Med Mol Imaging 29: 1149-1154, 2002.

16. Boellaard R, O'Doherty MJ, Weber WA, et al: FDG PET and PET/CT: EANM procedure guidelines for tumour PET imaging: version 1.0. Eur J Nucl Med Mol Imaging 37: 181-200, 2010.
17. Brenner W, Bohuslavizki KH and Eary JF: PET imaging of osteosarcoma. J Nucl Med 44: 930-942, 2003.

18. Gurney J, Swensen AR and Bulterys M: Malignant bone tumors. National Cancer Institute SEER Pediatric Monograph. ICCC VIII. National Cancer Institute, Bethesda, USA, pp99-110, 1999 .

19. Glasser DB, Lane JM, Huvos AG, Marcove RC and Rosen G: Survival, prognosis, and therapeutic response in osteogenic sarcoma: The Memorial Hospital experience. Cancer 69: 698-708, 1992.

20. Bacci G, Ferrari S, Bertoni F, et al: Long-term outcome for patients with nonmetastatic osteosarcoma of the extremity treated at the istituto orthopedic rizzoli according to the istituto ortopedico rizzoli/osteosarcoma-2 protocol: an updated report. J Clin Oncol 18: 4016-4027, 2000.

21. Cistaro A, Lopci E, Gastaldo L, et al: The role of 18F-FDG PET/CT in the metabolic characterization of lung nodules in pediatric patients with bone sarcoma. Pediatr Blood Cancer 59: 1206-1210, 2012.

22. Fletcher JW, Djulbegovic B, Soares HP, et al: Recommendations on the use of 18F-FDG PET in oncology. J Nucl Med 49: 480-508, 2008.

23. Erdi YE: The use of PET for radiotherapy. Curr Med Imaging Rev 3: 3-16, 2007

24. de Geus-Oei LF, van der Heijden HF, Corstens FH and Oyen WJ: Predictive and prognostic value of FDG-PET in nonsmall-cell lung cancer: a systematic review. Cancer 110: 1654-1664, 2007.

25. Lan XL, Zhang YX, Wu ZJ, et al: The value of dual time point (18)F-FDG PET imaging for the differentiation between malignant and benign lesions. Clin Radiol 63: 756-764, 2008.

26. Xiu Y, Bhutani C, Dhurairaj T, et al: Dual-time point FDG PET imaging in the evaluation of pulmonary nodules with minimally increased metabolic activity. Clin Nucl Med 32: 101-105, 2007.

27. Sahlmann CO, Siefker U, Lehmann K and Meller J: Dual time point 2-[18F]fluoro-2'-deoxyglucose positron emission tomography in chronic bacterial osteomyelitis. Nucl Med Commun 25: 819-823, 2004.

28. Johnson GR, Zhuang H, Khan J, et al: Role of positron emission tomography with fluorine-18-deoxyglucose in the detection of local recurrent and distant metastatic sarcoma. Clin Nucl Med 28: 815-820, 2003.

29. Klaeser B, Mueller MD, Schmid RA, Guevara C, Krause T and Wiskirchen J: PET-CT-guided interventions in the management of FDG-positive lesions in patients suffering from solid malignancies: initial experiences. Eur Radiol 19: 1780-1785, 2009.

30. Im HJ, Kim TS, Park SY, et al: Prediction of tumour necrosis fractions using metabolic and volumetric 18F-FDG PET/CT indices, after one course and at the completion of neoadjuvant chemotherapy, in children and young adults with osteosarcoma. Eur J Nucl Med Mol Imaging 39: 39-49, 2012.

31. Bajpai J, Gamnagatti S, Kumar R, et al: Role of MR imaging in osteosarcoma for evaluation and prediction of chemotherapy response: correlation with histological necrosis. Pediatr Radiol 41: 441-450, 2011.

32. Söderlund V, Larsson SA, Bauer HC, et al: Use of 99mTc-MIBI scintigraphy in the evaluation of the response of osteosarcoma to chemotherapy. Eur J Nucl Med 24: 511-515, 1997.

33. Bajpai J, Kumar R, Sreenivas V, et al: Prediction of chemotherapy response by PET-CT in osteosarcoma: correlation with histologic necrosis. J Pediatr Hematol Oncol 33: e271-e278, 2011.

34. Hawkins DS, Rajendran JG, Conrad EU III, Bruckner JD and Eary JF: Evaluation of chemotherapy response in pediatric bone sarcomas by [F-18]-fluorodeoxy-D-glucose positron emission tomography. Cancer 94: 3277-3284, 2002.

35. Sato J, Yanagawa T, Dobashi Y, Yamaji T, Takagishi K and Watanabe H: Prognostic significance of 18 F-FDG uptake in primary osteosarcoma after but not before chemotherapy: a possible association with autocrine motility factor/phospho-glucose isomerase expression. Clin Exp Metastasis 25: 427-435, 2008.

36. Walter F, Federman N, Apichairuk W, et al: 18F-fluorodeoxyglucose uptake of bone and soft tissue sarcomas in pediatric patients. Pediatr Hematol Oncol 28: 579-587, 2011.

37. Costelloe CM, Raymond AK, Fitzgerald NE, Mawlawi OR, Nunez RF, et al: Tumor necrosis in osteosarcoma: inclusion of the point of greatest metabolic activity from F-18 FDG PET/CT in the histopathologic analysis. Skeletal Radiol 39: 131-140, 2010. 
38. Fischer BM, Mortensen J, Langer SW, Loft A, Berthelsen AK, Petersen BI, et al: A prospective study of PET/CT in initial staging of small-cell lung cancer: comparison with CT, bone scintigraphy and bone marrow analysis. Ann Oncol 18: 338-345, 2007.

39. Kut V, Spies W, Spies S, Gooding W and Argiris A: Staging and monitoring of small cell lung cancer using [18F]fluoro-2-deox y-D-glucose-positron emission tomography (FDG-PET). Am J Clin Oncol 30: 45-50, 2007.

40. Erlemann R: MRI is highly sensitive for the detection of bone marrow abnormalities, cortical destruction or soft tissue tumors adjacent or infiltrating neighboring bones. Eur J Radiol 58: 48-67, 2006.

41. Antoch G, Kanja J, Bauer S, Kuehl H, Renzing-Koehler K, et al: Comparison of PET, CT, and dual-modality PET/CT imaging for monitoring of imatinib (STI571) therapy in patients with gastrointestinal stromal tumors. J Nucl Med 45: 357-365, 2004.

42. Barker JM and Silvestri GA: Lung cancer staging. Curr Opin Pulm Med 8: 287-293, 2002.

43. Gupta NC, Graeber GM and Bishop HA: Comparative efficacy of positron emission tomography with fluorodeoxyglucose in evaluation of small $(<1 \mathrm{~cm})$, intermediate $(1$ to $3 \mathrm{~cm})$, and large $(>3 \mathrm{~cm})$ lymph node lesions. Chest 117: 773-778, 2000.

44. Yi CA, Lee KS, Kim BT, et al: Tissue characterization of solitary pulmonary nodule: comparative study between helical dynamic CT and integrated PET/CT. J Nucl Med 47: 443-450, 2006.

45. Tateishi U, Yamaguchi U, Seki K, et al: Bone and soft-tissue sarcoma: preoperative staging with fluorine 18 fluorodeoxyglucose PET/CT and conventional imaging. Radiology 245 839-847, 2007.
46. Huang TL, Liu RS, Chen TH, et al: Comparison between F-18-FDG positron emission tomography and histology for the assessment of tumor necrosis rates in primary osteosarcoma. J Chin Med Assoc 69: 372-376, 2006

47. Kim JW, Kim HC, Park JW, et al: Predictive value of (18)FDG PET-CT for tumour response in patients with locally advanced rectal cancer treated by preoperative chemoradiotherapy. Int J Colorectal Dis. 28: 1217-1224, 2013.

48. Saifuddin A: The accuracy of imaging in the local staging of appendicular osteosarcoma. Skeletal Radiol 31: 191-201, 2002.

49. Drzezga A, Souvatzoglou M, Eiber M, et al: First clinical experience with integrated whole-body PET/MR: comparison to PET/CT in patients with oncologic diagnoses. J Nucl Med 53: $845-855,2012$.

50. Buchbender C, Heusner TA, Lauenstein TC, Bockisch A and Antoch G: Oncologic PET/MRI, part 1: tumors of the brain, head and neck, chest, abdomen, and pelvis. J Nucl Med 53: 928-938, 2012.

51. Cheon GJ, Kim MS, Lee JA, Lee SY, Cho WH, et al: Prediction model of chemotherapy response in osteosarcoma by $18 \mathrm{~F}-\mathrm{FDG}$ PET and MRI. J Nucl Med 50: 1435-1440, 2009.

52. Strobel K, Exner UE, Stumpe KD, et al: The additional value of CT images interpretation in the differential diagnosis of benign vs. malignant primary bone lesions with 18 F-FDG-PET/CT. Eur J Nucl Med Mol Imaging 35: 2000-2008, 2008. 